\title{
Benzoylation of $p$-Xylene at the Solid-Liquid Interface on Iron Incorporated Silica
}

\author{
Farook Adam*, Ishraga Abdelmoniem Hassan and Radhika Thankappan
}

School of Chemical Sciences, Universiti Sains Malaysia, 11800 Penang, Malaysia

\begin{abstract}
A series of iron incorporated silica catalysts were prepared using rice husk ash as a source of silica. The specific surface areas of the catalysts were found to be in the range of $200-400 \mathrm{~m}^{2} \mathrm{~g}^{-1}$. The activities of the catalysts were tested for liquid phase Friedel-Crafts acylation reaction of $p$-xylene with benzoyl chloride. The catalyst RHA-10Fe exhibited the highest activity for benzoylation of $p$-xylene. The conversion of benzoyl chloride $(\mathrm{BzCl})$ and the selectivity towards 2,5-dimethylbenzophenone (2,5-DMBP) were found to be 98.4 and 88.9 (\%) respectively at $413 \mathrm{~K}$.
\end{abstract}

Keywords: Rice husk silica, Friedel-Crafts acylation, benzoylation, $p$-xylene.

\section{INTRODUCTION}

The Friedel-Crafts acylation of aromatics is the main route for the formation of aromatic ketones in organic synthesis. These aromatic ketones are widely used for the production of pharmaceuticals, fragrances, flavours, insecticides and other products [1]. Acylation of $p$-xylene with benzoyl chloride yields 2,5-dimethylbenzophenone, which is used extensively as a UV light stabilizer in plastics, cosmetics and films [2]. Generally, these reactions are catalyzed by homogenous Lewis acids such as $\mathrm{AlCl}_{3}, \mathrm{BF}_{3}, \mathrm{FeCl}_{3}, \mathrm{ZnCl}_{2}$ or strong protonic acids (e.g. $\mathrm{H}_{2} \mathrm{SO}_{4}, \mathrm{HF}$ ) [3]. However, these homogenous catalysts have several problems, such as difficultly in separation and recovery, corrosion, high toxicity, requirements of more than stoichiometric amounts and the formation of many side products [4]. The application of homogenous catalysts generates a high volume of waste material. With the current drive towards green chemistry, it is recognized that there is a need to replace the conventional Lewis acid catalysts with reusable, environmentally friendly catalysts to produce minimal waste and by-products [5]. Recently, considerable efforts have been made to achieve this goal using a number of heterogeneous solid acid catalysts such as salts of heteropolyacids [6], zeolites [5, 7, 8], clays [2], sulphated $\mathrm{ZrO}_{2}$ [9] and mesoporous molecular sieves [10].

The benzoylation of aromatic compounds over zeolites and modified zeolites has been reported in the literature [5,8]. Suja et al. [9] studied the benzoylation of arenes over iron promoted sulphated zirconia. The benzoylation of substituted benzenes by benzoyl chloride over $\operatorname{In}_{2} \mathrm{O}_{3}(20 \%) / \mathrm{Si}$ MCM-41[10] showed a reactivity of about $82 \%$ for $p$-xylene within $8 \mathrm{~h}$. Yadav et al. [2] studied the benzoylation of $p$ xylene with benzoyl chloride using 20\% w/w cesiumsubstituted dodecatungstophosphoric acid supported on K-10

*Address correspondence to this author at the School of Chemical Sciences, Universiti Sains Malaysia, 11800 Penang, Malaysia;

Tel: +6046533567; Fax: +6046574854;

E-mail: farook@usm.my, farook_dr@yahoo.com clay catalyst. The conversion was $60 \%$ with $1.8 \mathrm{~g}$ of the catalyst at $403 \mathrm{~K}$ for $4 \mathrm{~h}$.

Rice husk (RH) is a major waste product of the rice milling industry. It is well known that the RH contains $20 \%$ of ash and the silica content of this ash is more than 95\% [11]. Amorphous silica of high surface area could be prepared from rice husk ash (RHA) [12]. Recently, there has been great interest for the use of rice husk silica as a support for preparing improved heterogeneous catalysts and adsorbents [13-17]. Iron incorporated silica from rice husk ash was found to be active in the Friedel-Crafts benzylation of toluene with high product yield of more than 92\% [18]. Adam et al. [19] reported the synthesis of 4-(methylamino)benzoic acid incorporated iron-rice husk silica for benzylation of toluene with the selective formation of monobenzyl toluene. The iron-silica heterogeneous catalysts were also used for the benzylation of benzene and xylene [20,21].

In this paper, we report the preparation, characterization and catalytic activity of iron loaded silica catalysts from RHA in the benzoylation of $p$-xylene. To the best of our knowledge, the benzoylation of $p$-xylene over iron loaded rice husk silica catalyst for producing 2,5-dimethybenzophenone has not been studied.

\section{EXPERIMENTAL}

\section{Materials}

Rice husk was collected from a local rice mill in Penang. The chemicals used were sodium hydroxide (Systerm, 99\%), nitric acid (Systerm, 65\%), iron nitrate-nonahydrate $\mathrm{Fe}\left(\mathrm{NO}_{3}\right)_{3} \cdot 9 \mathrm{H}_{2} \mathrm{O}$ (Systerm, 98\%), benzoyl chloride (Fluka, 98\%), $p$-xylene (Fluka, $\geq 98 \%$ ), and decane (Acros-organics, 99\%). All the chemicals were of AR grade and used as received.

\section{Catalyst Preparation}

The treatment of $\mathrm{RH}$ and the preparation of catalysts were carried out according to literature [22]. A $5.0 \mathrm{~g}$ sample of the treated RHA was dissolved in $250 \mathrm{~mL}$ of $1.0 \mathrm{M}$ 
$\mathrm{NaOH}$, stirred for $18 \mathrm{~h}$ at room temperature and filtered to yield a sodium silicate solution. To prepare 5, 10, 15 and 20 wt.\% Fe incorporated catalyst, masses of 1.81, 3.62, 5.43 and $7.23 \mathrm{~g}$ respectively of $\mathrm{Fe}\left(\mathrm{NO}_{3}\right)_{3} .9 \mathrm{H}_{2} \mathrm{O}$ were dissolved in 3.0 $\mathrm{M} \mathrm{HNO}_{3}$ and this solution was titrated against sodium silicate solution until $\mathrm{pH}$ 5.0. The gel formed was separated by centrifuge (Model T 30, JANETZKI), washed with distilled water followed by acetone and dried at $110{ }^{\circ} \mathrm{C}$ for $24 \mathrm{~h}$. The catalysts were denoted as RHA-xFe where $\mathrm{x}=5,10,15$ and 20 wt.\% Fe.

\section{THE CATALYSTS CHARACTERIZATION}

The prepared catalysts were characterized by powder Xray diffraction (Siemens Diffractometer D5000, Kristalloflex), FT-IR spectroscopy (Perkin Elmer system 2000), $\mathrm{BJH} \mathrm{N}_{2}$-sorption analysis (Micromeritics Instrument Corporation Model ASAP 2000), diffuse reflectance UV-Vis (Perkin Elmer Lambda $35 \mathrm{UV}-\mathrm{Vis}$ spectrometer), energy dispersive spectrometry (Oxford INCA 400), inductively coupled plasma atomic emission spectrometer (PE SCIEX ELAN 6100) and scanning electron microscopy (Leo Supra $50 \mathrm{VP})$. The acidity of the catalysts was studied by pyridine adsorption using the following procedure: about $0.05 \mathrm{~g}$ of the catalyst was kept with pyridine in a desiccator equipped with a valve connected to a membrane vacuum pump (AMB Greiffenberger Antriebstechnik, MZ2C, CE 2002/06). The system was evacuated for $1 \mathrm{~h}$ at a rate of $1.7 \mathrm{~m}^{3} \mathrm{~h}^{-1}$ and kept under closed vacuum for $48 \mathrm{~h}$. The excess pyridine in the desiccator was evacuated again for $1 \mathrm{~h}$ at the same pump rate. The catalyst was removed and analyzed by FT-IR in the range of $1700-1400 \mathrm{~cm}^{-1}$.

\section{BENZOYLATION OF $p$-XYLENE WITH BENZOYL CHLORIDE}

The liquid phase benzoylation of $p$-xylene ( $p$-xyl) with benzoyl chloride $(\mathrm{BzCl})$ was carried out in a $50 \mathrm{~mL}$ twonecked round bottom flask equipped with a reflux condenser. The temperature of the system was controlled using a thermocouple in an oil bath. A constant flow of dry argon was maintained to minimize the effect of moisture throughout the reaction period.

In a typical reaction, $p$-xyl $(189.9 \mathrm{mmol})$ was added to $0.5 \mathrm{~g}$ of the activated catalyst $(383 \mathrm{~K})$ in a round bottom flask and kept in an oil bath. After attaining the reaction temperature, $\mathrm{BzCl}(9.3 \mathrm{mmol})$ was added and stirred magnetically $(600 \mathrm{rpm})$. Decane was added into the reaction system as an internal standard. The products were withdrawn at regular intervals and analyzed on gas chromatography (Clarus 500, Perkin Elmer) equipped with flame ionization detector and capillary column (Elite-5, $30 \mathrm{~m}$ length and $0.32 \mathrm{~mm}$ inner diameter). The set up temperature program was: initial oven temperature- $90{ }^{\circ} \mathrm{C}$, heating rate $10{ }^{\circ} \mathrm{C} \mathrm{min}^{-1}$, and final temperature-220 ${ }^{\circ} \mathrm{C}$. The detector and injector temperatures were maintained as $250{ }^{\circ} \mathrm{C}$.

In order to check the reusability of catalysts, the used catalyst was allowed to settle in the reaction system. After removing the supernatant mixture, fresh reagents were added to continue the reaction [4].

To test of metal leaching, the reaction mixture was separated after 4 min with a micro membrane filter. The reaction mixture was transferred into a preheated empty reaction vessel to continue the reaction. The products were monitored as before.

\section{RESULTS AND DISCUSSION}

\section{Powder X-ray Diffraction}

The powder X-ray diffraction (XRD) patterns of the RHA-SiO ${ }_{2}$ and all Fe loaded silica catalysts are shown in Fig. 1. The broad pattern observed at $2 \theta=23^{\circ}$ corresponds to the amorphous nature of the silica [23, 24]. All catalysts appear amorphous without any crystalline nature. When the Fe content increased, the peak intensity decreased indicating that the addition of $\mathrm{Fe}$ has an influence on the amorphous nature of the silica.

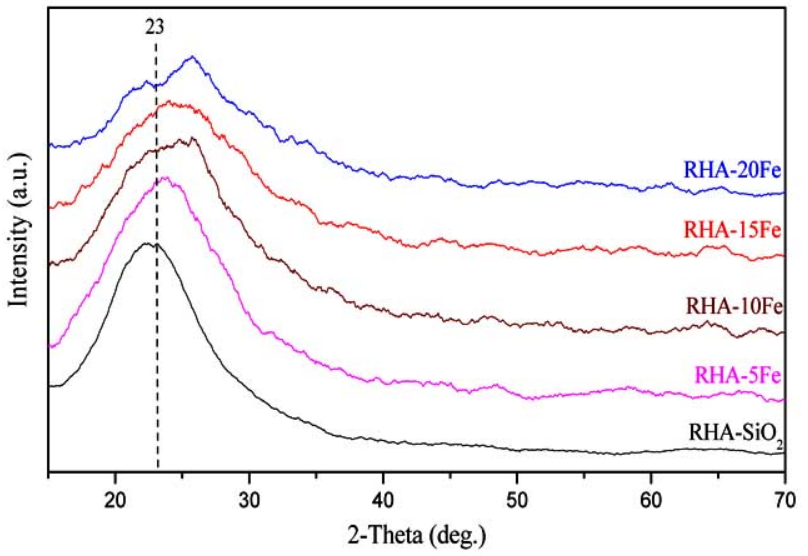

Fig. (1). The powder X-ray diffraction pattern of the prepared catalysts.

\section{FT-IR Spectra}

The FT-IR spectra of the RHA-SiO 2 and various $\mathrm{Fe}$ loaded silica catalysts are shown in Fig. 2. In $\mathrm{RHA}-\mathrm{SiO}_{2}$, the strong band at $1096 \mathrm{~cm}^{-1}$ is due to the asymmetric stretching vibration of the structural siloxane bond, Si-O-Si [25-28]. Upon Fe loading, an observed shift of this band to lower wavenumber was detected, possibly due to the formation of $\mathrm{Si}-\mathrm{O}-\mathrm{Fe}$ bonds. The band at $976 \mathrm{~cm}^{-1}$ in $\mathrm{RHA}-\mathrm{SiO}_{2}$ is attributed to $\mathrm{Si}-\mathrm{OH}$ asymmetric stretching vibration. This band appears as a shoulder at $972 \mathrm{~cm}^{-1}$ in RHA-5Fe and completely vanished as the Fe loading was increased. This could be due to the increased presence of $\mathrm{Si}-\mathrm{O}-\mathrm{Fe}$ bonds at the expense of surface $\mathrm{Si}-\mathrm{OH}[21,29]$.

\section{Nitrogen-Sorption Analysis}

The $\mathrm{N}_{2}$ adsorption-desorption analysis results obtained for the catalysts are presented in Fig. 3. In all cases, the isotherms were of type IV, which is characteristic of mesoporous materials according to IUPAC classification [30]. The pure silica exhibited IUPAC type $\mathrm{H} 2$ hysteresis loop, which is characteristic of corpuscular systems consisting of the distribution of pore size and shape is not well defined. RHA-5Fe exhibited H1 hysteresis loop, which is characteristic of porous material consisting of agglomerates or compacts of spheroidal particles with uniform size and array occur in the curve at a relative pressure of $0.5<\mathrm{P} / \mathrm{P}_{0}<0.9$ resulting from the capillary condensation and desorption of $\mathrm{N}_{2}$, which strongly suggest the presence of large mesopores [31]. When the Fe content increased from 10 to $20 \mathrm{wt} \%$, the hysteresis loop transforms gradually from type $\mathrm{H} 1$ to type 
H3 suggesting the presence of slit shaped pores with non-uniform size and shape. The inflection point of $\mathrm{P} / \mathrm{P}_{0}=1$ indicates that the presence of large mesopores arising from textural mesoporosity and gives non homogenous pore size distribution [32].

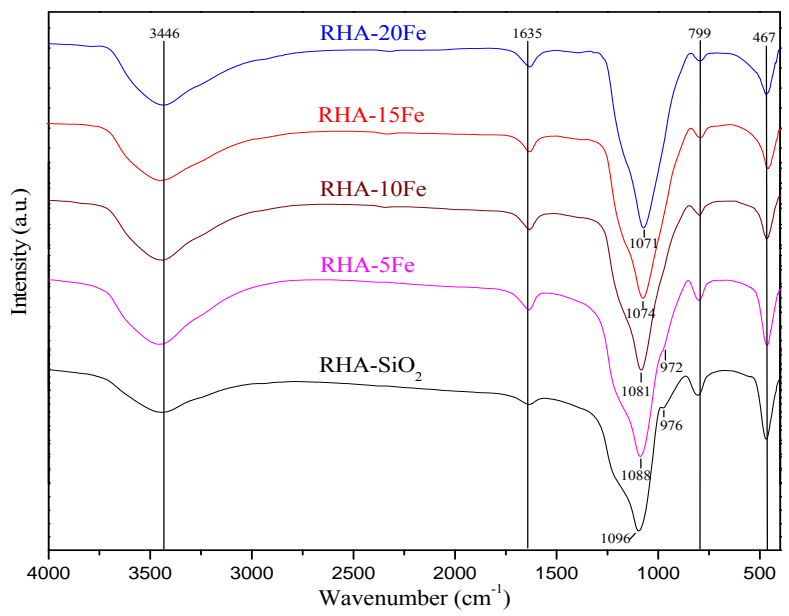

Fig. (2). The FT-IR spectra of the catalysts.

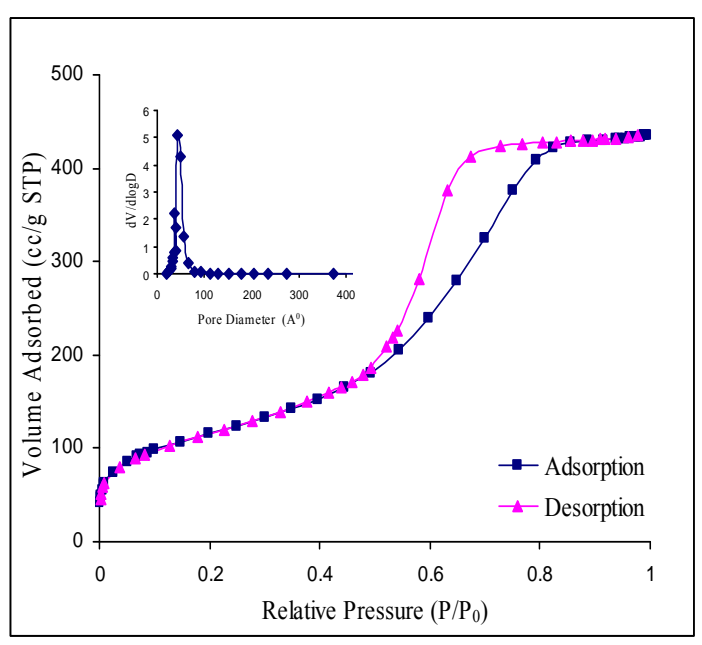

(a)

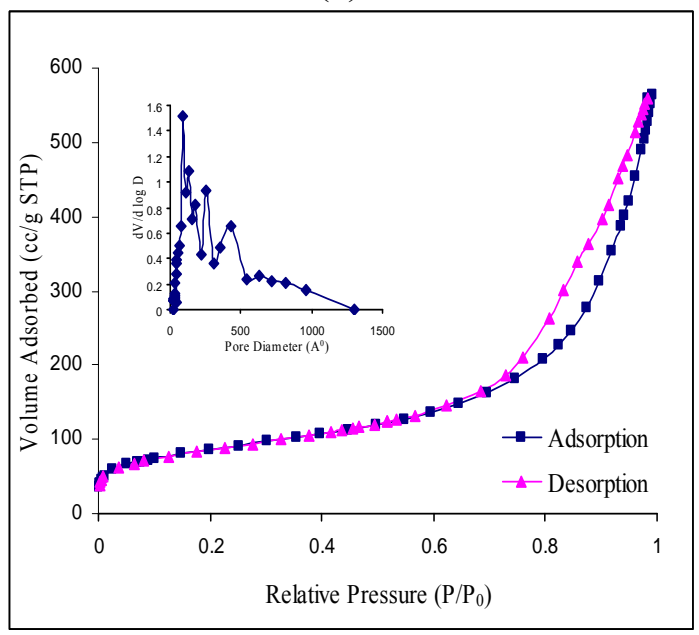

(c)

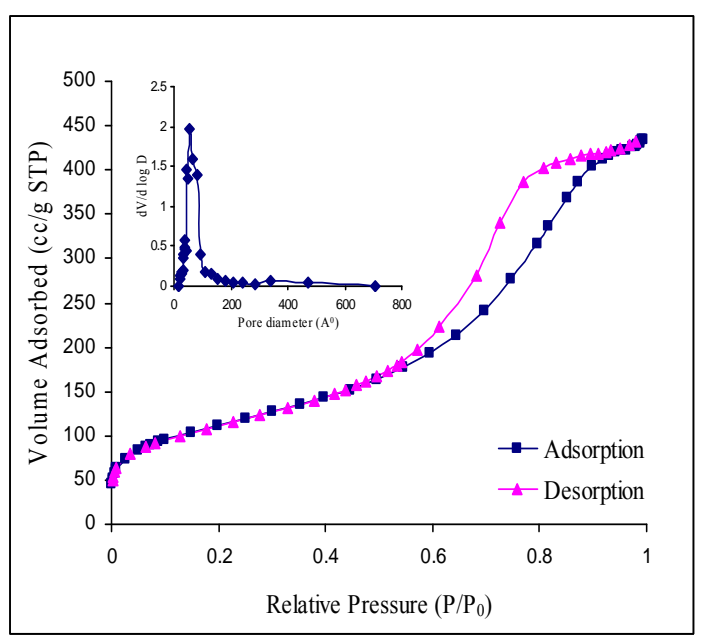

(b)

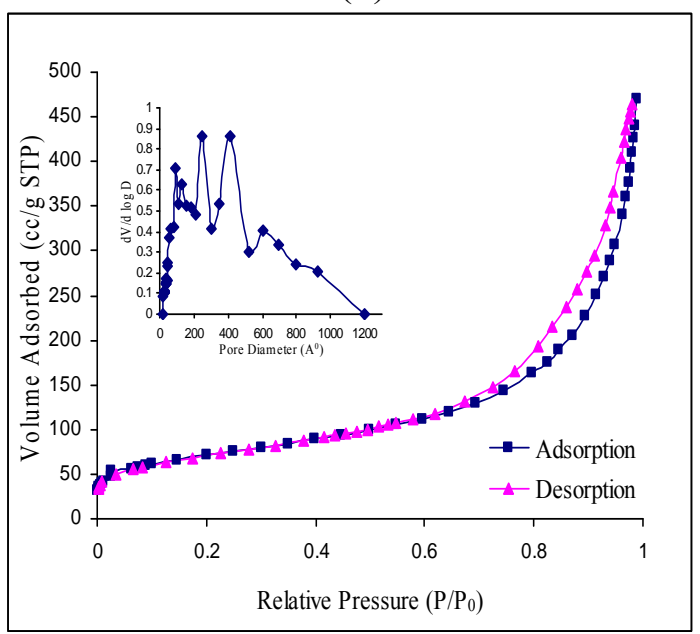

(d) 
Fig. 3. contd....

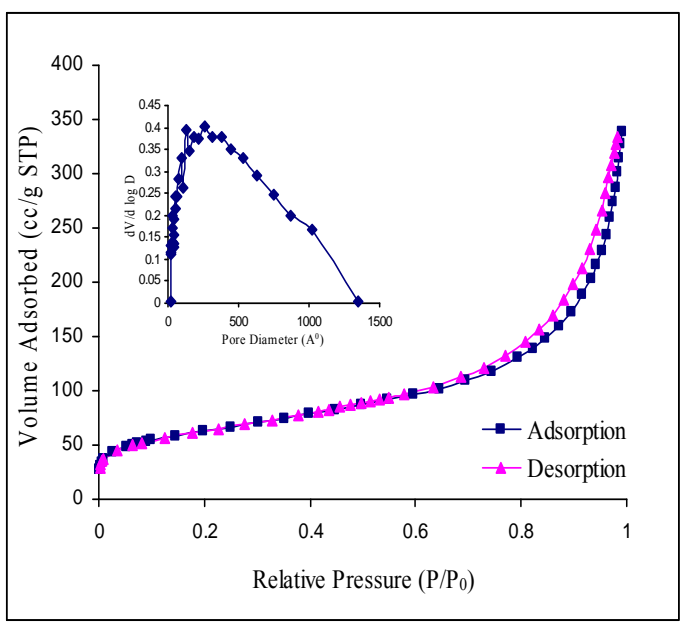

(e)

Fig. (3). Nitrogen adsorption-desorption isotherms and the corresponding pore size distribution of (a) $\mathrm{RHA}^{-\mathrm{SiO}} 2$ (b) $\mathrm{RHA}^{-5 F e}(\mathbf{c}) \mathrm{RHA}-10$ $\mathrm{Fe}$ (d) RHA-15Fe and (e) RHA-20Fe.

Textural parameters of the prepared catalysts obtained from $\mathrm{N}_{2}$-sorption analysis is presented in Table 1. The prepared $\mathrm{RHA}-\mathrm{SiO}_{2}$ was found to have a surface area of $414 \mathrm{~m}^{2}$ $\mathrm{g}^{-1}$. As the iron loading increased from 5-20 wt.\%, a gradual decrease in the specific surface area was observed. The decrease in the surface area could be due to agglomeration of the silica-Fe particles as the metal loading was increased $[9,33]$. The average pore diameter increased upon Fe loading to $10 \mathrm{wt} . \%$ (RHA-10Fe) and subsequently decreased upon further increase in $\mathrm{Fe}$.

\section{Diffuse Reflectance UV-Visible Spectroscopy}

The DR UV-Vis spectra of the catalysts are shown in Fig. 4. The spectra in all catalysts show absorption bands at 245 $\mathrm{nm}$ corresponding to oxygen to metal charge transfers involving isolated tetrahedral coordinated $\mathrm{Fe}^{3+}$ [34]. The absence of bands above $320 \mathrm{~nm}$ confirmed that no clustered $\mathrm{Fe}_{2} \mathrm{O}_{3}$ or $\mathrm{Fe}_{3} \mathrm{O}_{4}$ species were present in the prepared catalysts [35].

\section{Elemental Analyses}

The elemental analyses result of the RHA- $\mathrm{SiO}_{2}$ and various Fe loaded silica catalysts carried out by EDX (surface analysis) and ICP-MS (which involves complete dissolution of the sample prior to analysis) are shown in Table 2. The results show the presence of $\mathrm{Fe}^{3+}$ ions in the $\mathrm{Fe}$ loaded silica

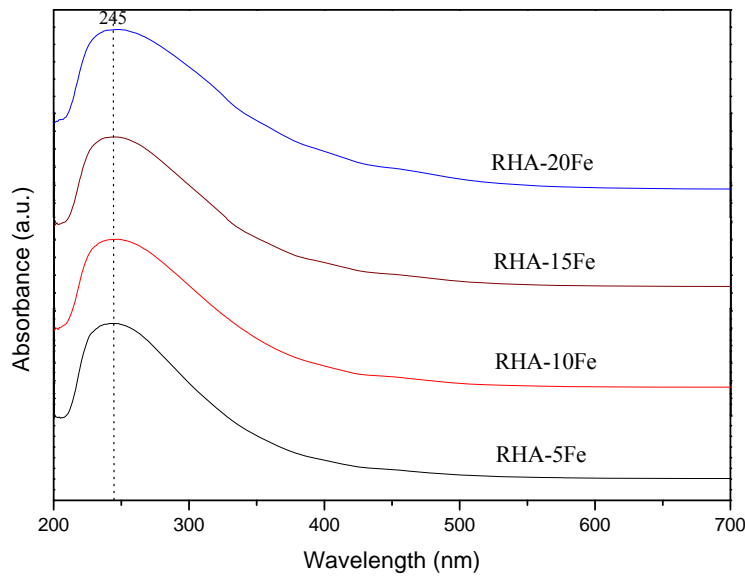

Fig. (4). DR UV/Vis spectra of prepared catalysts.

samples, while it was completely absent in RHA-SiO ${ }_{2}$ sample. The amount of Fe detected by EDX and ICP-MS are in good agreement with each other. The incorporated Fe was found to be lower than the actual amount used for the preparation of the catalyst. This could arise from the tension generated within the silicate structure due to the difference in the

Table 1. The Textural Parameters of Prepared Catalysts

\begin{tabular}{|c|c|c|}
\hline Catalyst & BET Surface area $\left(\mathbf{m}^{2} \mathbf{g}^{-1}\right)$ & Average Pore Diameter (nm) \\
\hline \hline RHA-SiO & 6.48 \\
\hline RHA-5Fe & 414 & 6.75 \\
\hline RHA-10Fe & 393 & 10.6 \\
\hline RHA-15Fe & 301 & 9.80 \\
\hline RHA-20Fe & 248 & 8.15 \\
\hline
\end{tabular}


ionic radii of $\mathrm{Fe}^{3+}$ and $\mathrm{Si}^{4+}$ bond lengths resulting in distorted silica structures. This can limit the amount of Fe to be incorporated within the silica structure [36].

\section{Acidity of the Catalysts}

The FT-IR spectra obtained after pyridine adsorption are shown in Fig. 5. The strong band at about $1638 \mathrm{~cm}^{-1}$ can be assigned to the stretching vibration of pyridinium ion that binds to Brønsted as well as Lewis acid sites [37]. Characteristic bands found from 1551 to $1565 \mathrm{~cm}^{-1}$ are assigned to Brønsted acid sites and the band at $1450 \mathrm{~cm}^{-1}$ can be attributed to strong Lewis acid sites [38,39]. The additional band at $1491 \mathrm{~cm}^{-1}$ in RHA-10Fe is assigned to pyridine associated with both Brønsted and Lewis acid sites [38]. All the prepared catalysts contain both Brønsted and Lewis acid sites, which can make the benzoylation reaction more feasible.

\section{Microscopy Analysis}

Fig. 6 shows the SEM backscattered electron micrographs of $\mathrm{RHA}-\mathrm{SiO}_{2}$ and $\mathrm{Fe}$ loaded silica catalysts. The SEM image of RHA-SiO 2 shows agglomerates of spherical particles. The surface of RHA-5Fe was composed of irregular shape particles. As iron loading increased, these particles are stick together to form agglomerates [36].

\section{CATALYTIC ACTIVITY OF VARIOUS CATALYSTS}

To optimize the reaction parameters, the benzoylation reaction was carried out using $\mathrm{RHA}-10 \mathrm{Fe}$ as a representative catalyst by varying the reaction conditions such as, catalyst mass, molar ratio, temperature, etc.

\section{Effect of Catalyst Mass}

To determine the optimum mass of the catalyst, reactions were carried out by varying the mass of the catalyst from 0.1 to $0.5(\mathrm{~g})$. The results are presented in Table 3 . The conversion of $\mathrm{BzCl}$ was found to increase from 44.8 to $62.5 \%$ as the mass of the catalyst increased. This can result from the increase in the number of active sites available for the reaction [7]. The main product formed in the benzoylation of $p$ xyl was 2,5-DMBP with minor side products as: benzoic acid (BA), benzoic anhydride (BAN) and 2,4-DMBP.

Table 2. The Average Fe Content (w/w) \% Obtained by EDX and ICP-MS of Catalysts

\begin{tabular}{|l|c|c|}
\hline \multirow{2}{*}{ Catalysts } & \multicolumn{2}{|c|}{ Average Fe content (w/w)\% } \\
\cline { 2 - 4 } & EDX & - \\
\hline \hline RHA-SiO & - & 4.39 \\
\hline RHA- $5 \mathrm{Fe}$ & 3.54 & $7.22(4.96)^{*}$ \\
\hline RHA-10Fe & 7.58 & 10.46 \\
\hline RHA-15Fe & 9.91 & 11.95 \\
\hline RHA-20Fe & 13.90 & \multicolumn{2}{|c|}{} \\
\hline
\end{tabular}

*After first recycle experiment.

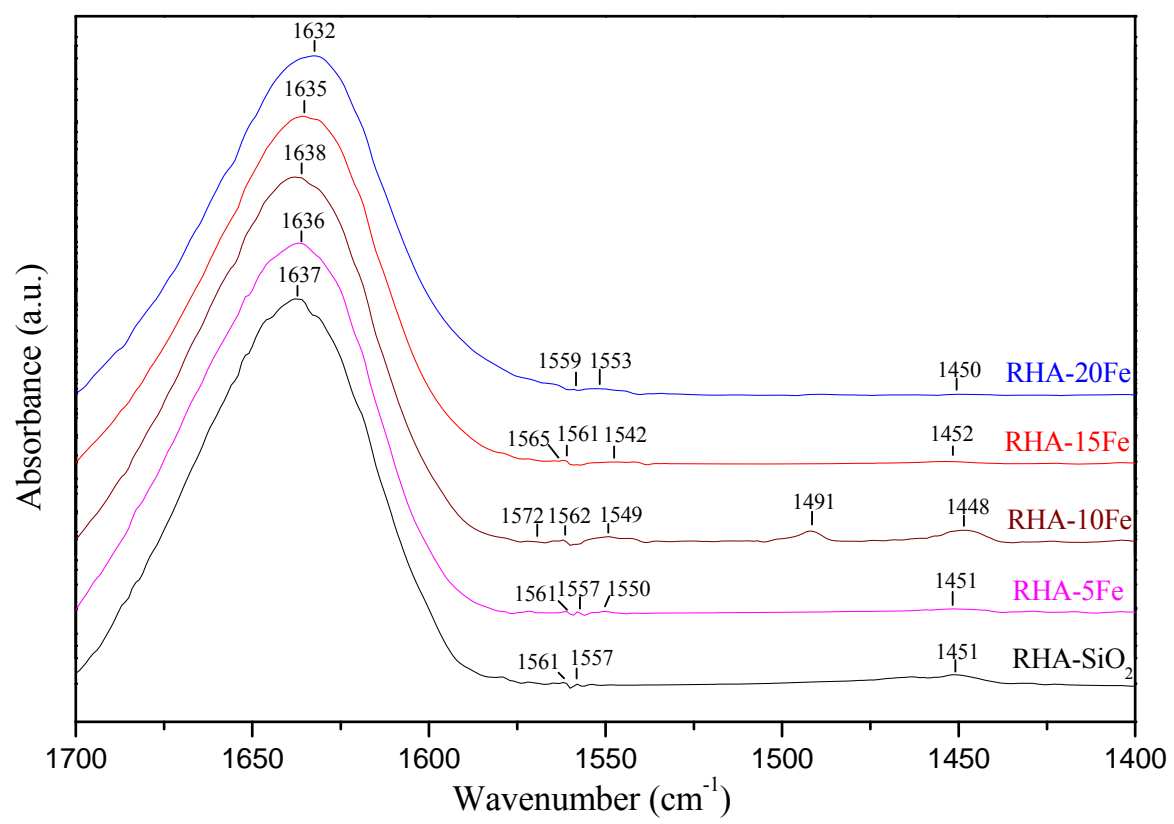

Fig. (5). The pyridine-FTIR spectra of RHA-SiO 2 , RHA-5Fe, RHA-10Fe, RHA-15Fe and RHA-20Fe at room temperature. 

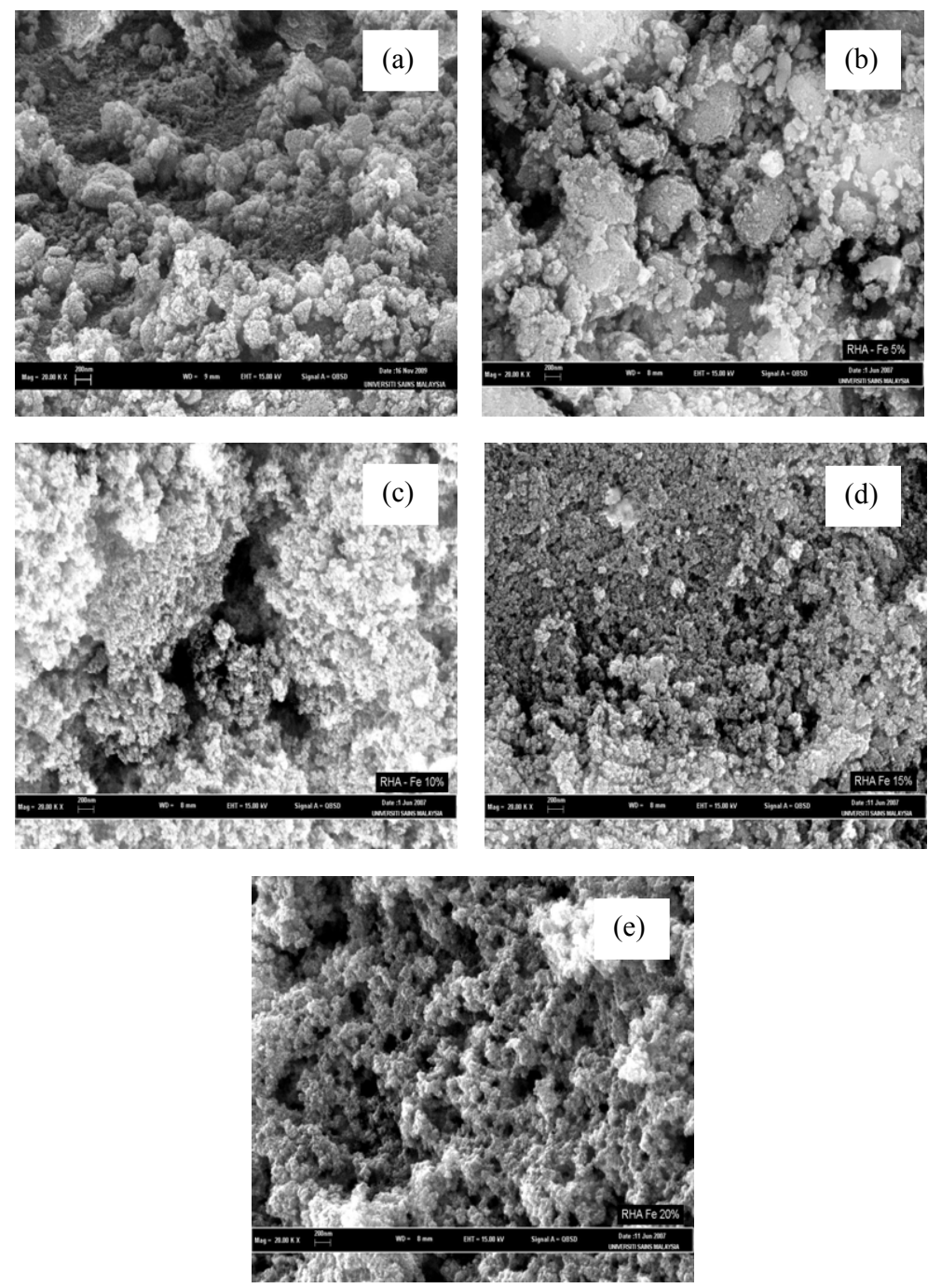

Fig. (6). The SEM images of (a) $\mathrm{RHA}^{-\mathrm{SiO}_{2}}$ (b) RHA-5Fe (c) RHA-10Fe (d) RHA-15Fe and (e) RHA-20Fe.

Table 3. Effect of Catalyst Mass on the Reaction

\begin{tabular}{|c|c|c|c|c|c|}
\hline \multirow{2}{*}{ Catalyst Mass (g) } & Conversion of BzCl (\%) & \multicolumn{4}{|c|}{ Selectivity (\%) } \\
\cline { 3 - 6 } & & BA & 2,5-DMBP & 2,4- DMBP & BAN \\
\hline \hline 0.1 & 44.8 & 11.1 & 80.1 & 0.7 & 0.5 \\
\hline 0.3 & 57.8 & 30.3 & 57.5 & 11.7 \\
\hline 0.5 & 62.5 & 15.9 & 80.1 & 0.7 & 3.3 \\
\hline
\end{tabular}

Reaction condition: Temperature $=353 \mathrm{~K}, \mathrm{BzCl}: p$-xyl $=1: 15$, catalyst $=\mathrm{RHA}-10 \mathrm{Fe}$, time $=9 \mathrm{~h}$.

\section{Influence of Benzoyl Chloride: $p$-Xylene Molar Ratio}

The influence of BzCl:p-xyl molar ratio on the conversion and product distribution over RHA- $10 \mathrm{Fe}$ at $353 \mathrm{~K}$ is shown in Fig. 7. As the molar ratio increased from 1:5 to $1: 20$, the $\mathrm{BzCl}$ conversion also increased. At a molar ratio of $1: 20$, high conversion of $\mathrm{BzCl}(86.0 \%)$ was observed. At the lower concentration of benzoyl chloride more active sites of catalyst are available for adsorption, which results in the formation of active electrophilic benzoylinium cation that can react with $p$-xyl. At lower mole ratios, the concentration of benzoyl chloride with respect to $p$-xyl increases and the active sites become saturated with benzoyl chloride, resulting in the formation of fewer active electrophilic benzoylinium ion to react with the $p$-xyl present in the bulk phase. Thereby showing a decrease in conversion of benzoyl chloride $[2,40]$. In addition, not much difference is observed for the selective formation of 2,5-DMBP as the molar ratio changed from 1:5 to 1:20. Thus the optimum molar ratio of 1:20 was used for further studies. 


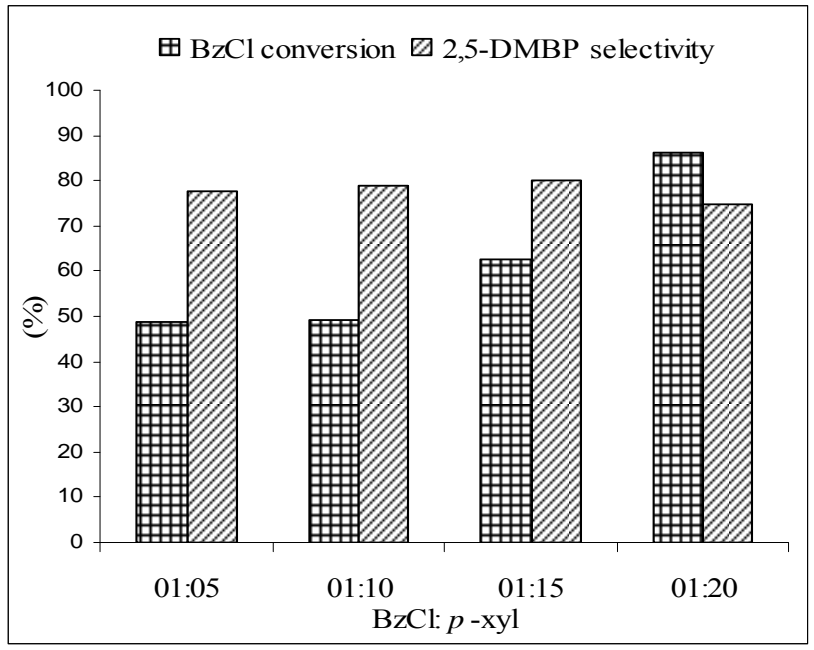

Fig. (7). Effect of molar ratios of BzCl:p-xyl over RHA-10Fe on the conversion of $\mathrm{BzCl}$ and selectivity towards 2,5-DMBP: Temperature $=353 \mathrm{~K}$, catalyst mass $=0.5 \mathrm{~g}$, time $=9 \mathrm{~h}$.

\section{Effect of Reaction Temperature}

In order to study the effect of temperature, the benzoylation was carried out from 353 to $413 \mathrm{~K}$ with 1:20 molar ratio (BzCl:p-xyl) over RHA-10Fe. The results are shown in Fig. 8. The conversion of $\mathrm{BzCl}$ was found to increase substantially with increasing reaction temperature. However, the time taken to reach the maximum conversion was shorter as the temperature increased. Selectivity to 2,5-DMBP also increased as the temperature increased. A conversion of $98.4 \%$ of $\mathrm{BzCl}$ was achieved over RHA-10Fe in $1 \mathrm{~h}$ with selectivity of $88.9 \%$ for 2,5 -DMBP at the reaction temperature of $413 \mathrm{~K}$. This can be attributed to the faster reaction which decreased the probability of forming side products due to rearrangements.

\section{Effect of Metal Loading}

The benzoylation reaction was carried out at optimized reaction conditions over various metals loaded catalysts. Reaction was also done with homogeneous iron salt for comparison. The results are shown in Table 4 . The activity of the catalysts under the optimized condition was found to increase as the metal loading increased from 5 to $10 \mathrm{wt} . \%$ (98.4\%, RHA-10Fe). However, further increase of metal loading to 15 and $20 \mathrm{wt} . \%$ did not have much effect on the activity. In addition, the selectivity to 2,5-DMBP was more for RHA- $10 \mathrm{Fe}(88.8 \%)$. As the Fe loading increased more than $10 \mathrm{wt} . \%$, a decrease in selective for 2,5-DMBP was observed. There was no reaction observed without catalyst or in the presence of RHA-SiO${ }_{2}$. Thus, it can be concluded that the catalyst support itself $\left(\mathrm{RHA}-\mathrm{SiO}_{2}\right)$ shows no catalytic activity in the benzoylation of $p$-xyl. However, upon incorporation of $\mathrm{Fe}$ onto silica, the catalytic system became active due to the incorporated $\mathrm{Fe}$ on the silica. The product selectivity was most likely due to the restrictions imposed by the pore size and structure.

The conversion rate was much faster $(8 \mathrm{~min})$ for the homogenous iron salt compared to the heterogeneous catalyst with $\mathrm{BzCl}$ conversion of $99.4 \%$. However, the selectivity for

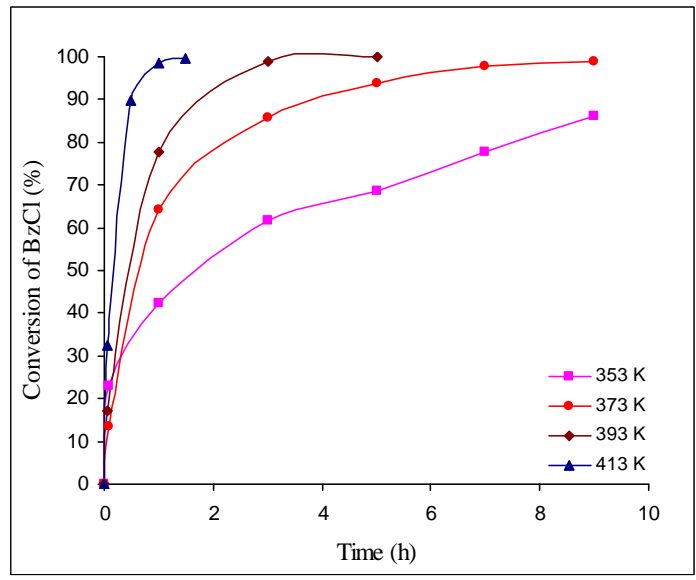

(a)

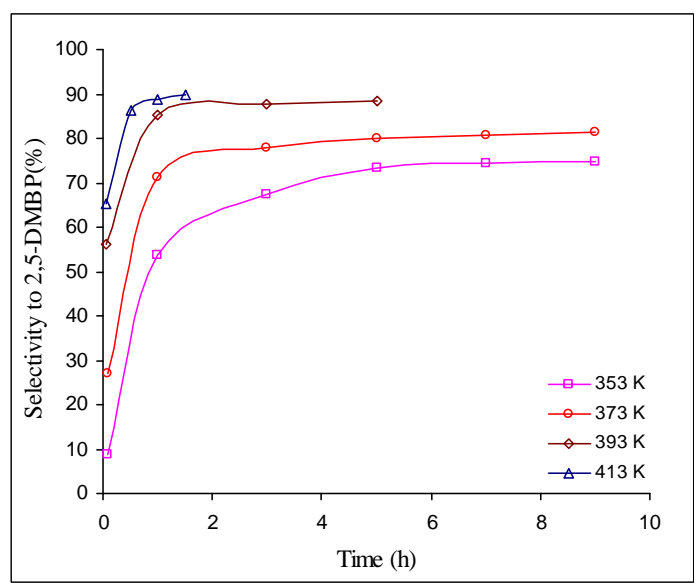

(b)

Fig. (8). The effect of reaction temperature on (a) the conversion of $\mathrm{BzCl}$ (b) the selectivity to 2,5-DMBP over RHA-10Fe: BzCl: $p$-xyl molar ratio $=1: 20$, catalyst mass $=0.5 \mathrm{~g}$.

2,5-DMBP was found to be only $50.4 \%$ for the homogeneous iron salt. A much higher selectivity for the desired product (2,5-DMBP) was afforded by the heterogeneous catalyst. Therefore, the heterogeneity of Fe on RHA-silica showed beneficial characteristics, especially for the selective formation of 2,5-DMBP.

The high benzoylation activity observed for these heterogeneous catalysts can be related to the presence of Brønsted and Lewis acid sites as observed from FT-IR-pyridine spectra (Fig. 5). It could also result from the high BET surface area. The redox properties of Fe supported systems can give rise to high benzoylation activity [9]. Decrease in the selective formation of 2,5-DMBP for RHA-15Fe and RHA$20 \mathrm{Fe}$ can be due to the decrease in surface area as well as the wide pore size (Fig. 3) [41].

\section{Stability and Reusability}

The reusability of the catalyst in the benzoylation of $p$ xyl was studied under the optimum conditions according to a published method [4]. The results are presented in Table 5. During its first reuse, the conversion of $\mathrm{BzCl}$ decreased from 
Table 4. Effect of Various Fe Loading

\begin{tabular}{|c|c|c|c|c|c|}
\hline \multirow{2}{*}{ Catalyst } & Conversion of BzCl $(\%)$ & \multicolumn{3}{|c|}{ Selectivity (\%) } \\
\cline { 3 - 6 } & & BA & 2,5-DMBP & 2,4- DMBP & 0.6 \\
\hline \hline RHA-5Fe & 77.7 & 10.1 & 88.5 & 0.8 \\
\hline RHA-10Fe & 98.4 & 10.3 & 88.8 & 0.6 & 0.5 \\
\hline RHA-15Fe & 97.5 & 14.7 & 84.6 & 0.2 \\
\hline RHA-20Fe & 98.7 & 16.0 & 83.3 & 0.2 \\
\hline $\mathrm{Fe}\left(\mathrm{NO}_{3}\right)_{3} .9 \mathrm{H}_{2} \mathrm{O}^{\mathrm{a}}$ & $99.4^{\mathrm{b}}$ & n.d. & 50.4 & n.d. \\
\hline
\end{tabular}

${ }^{\mathrm{a}}$; mass- $0.275 \mathrm{~g},{ }^{\mathrm{b}}$; time $=8 \mathrm{~min}$. Reaction conditions: Temperature $=413 \mathrm{~K}, \mathrm{BzCl}: p$-xyl molar ratio $=1: 20$, catalyst mass $=0.5 \mathrm{~g}$, time $=1 \mathrm{~h}$.

Table 5. Reusability of the Catalyst

\begin{tabular}{|c|c|c|c|c|c|}
\hline \multirow{2}{*}{ Cycles } & Conversion of BzCl (\%) & \multicolumn{4}{|c|}{ Selectivity (\%) } \\
\cline { 3 - 6 } & & BA & 2,5-DMBP & 2,4- DMBP & BAN \\
\hline \hline Fresh & 98.7 & 11.2 & 88.2 & 0.5 & 0.1 \\
\hline $1^{\text {st }}$ & 71.5 & 6.5 & 91.5 & 0.5 & 0.5 \\
\hline $2^{\text {nd }}$ & 42.4 & 4.1 & 86.8 & 8.6 \\
\hline
\end{tabular}

Reaction conditions: Temperature $=413 \mathrm{~K}, \mathrm{BzCl}: p$-xyl $=1: 20$, time $=1 \mathrm{~h}$, catalyst mass $=0.5 \mathrm{~g}$ of RHA-10Fe.

98.7 to $71.5 \%$. Further reuse reduced conversion to $42.4 \%$ suggesting leaching of some active metal centres during the reaction. However, the selectivity of 2,5-DMBP remain almost similar during all runs. The insignificant change in the selectivity of 2,5-DMBP and the noticeable decrease in the conversion suggest that there is a possible loss of Fe active sites from the catalyst. This was confirmed from ICP-MS analysis of the catalyst after the first recycle. The amount of Fe decreased from 7.22 to $4.96 \mathrm{w} / \mathrm{w} \%$ (Table 2), thus decreasing the catalytic activity. This could be due to the use of $\mathrm{BzCl}$ as the acylating agent, which is known to be corrosive. It could also be due to the presence of benzoic acid and the $\mathrm{HCl}$ (from the reaction) in the reaction mixture. According to Corma [42], leaching of active metal species can take place during the reaction depending on the nature of the substrate, the solvent and the reaction conditions. We are actively trying to overcome this leaching to improve the catalyst. We hope to report these improvements in the future.

\section{Leaching}

In order to investigate the details of the decreased activity observed in the previous section, a leaching test was carried out at $393 \mathrm{~K}$ since the reaction was so fast at the optimized temperature of $413 \mathrm{~K}$ (Fig. 9). After 4 minutes of reaction (13.8\% conversion of $\mathrm{BzCl}, 58.0 \%$ selectivity to 2,5DMBP), reactants were carefully withdrawn and filtered while hot and charged into an empty round bottom flask equipped with a condenser maintained at the same temperature. The system was allowed to react further without the catalyst. It can be seen that the conversion of $\mathrm{BzCl}$ continued even after removal of the catalyst. However, the selectivity towards 2,5-DMBP remains almost same (58.5\%) as in comparison to ca. $88 \%$ in the presence of the catalyst (Fig. 8). In addition, more amounts of BA and BAN were observed dur- ing the course of reaction. Increase in conversion indicates that leaching of $\mathrm{Fe}$ may take place during the reaction. Arends and Sheldon [43] had reported that the metal leaching is possible by the action of strongly corrosive products such as carboxylic acid or when using acetic acid as a solvent which leads to rapid leaching of the metal ions. Yadav et al. [40] had shown that $20 \%(\mathrm{w} / \mathrm{w})$ dodecatungstophosphoric acid on K-10 clay $(20 \%(w / w) ~ D T P / K-10)$ was more efficient for the acylation reaction when acetyl chloride was used as the acylating agent. However, when the highly corrosive acylating agent benzoyl chloride was used, the efficiency of the cata-

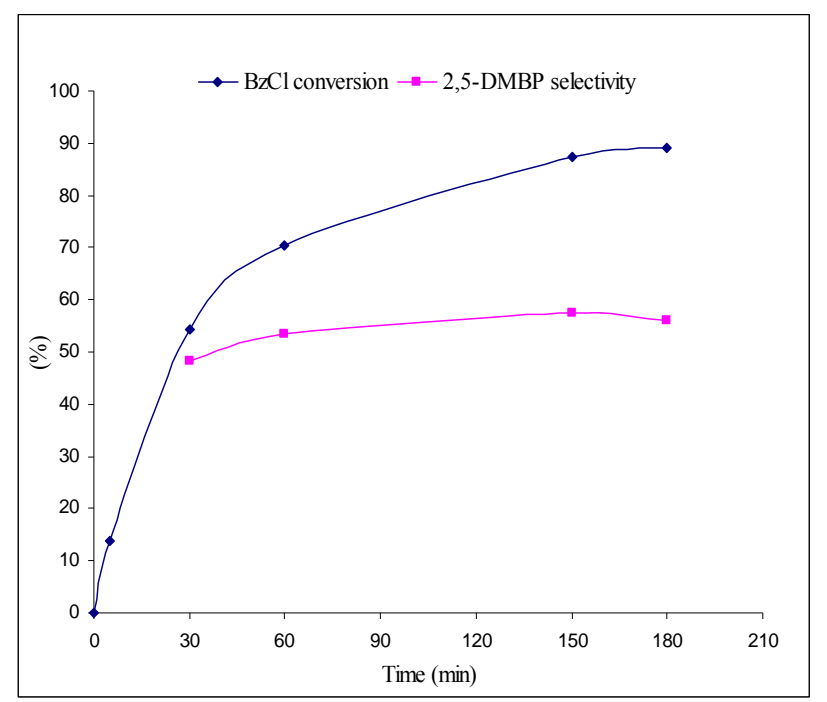

Fig. (9). The leaching effect over RHA-10Fe on the conversion of $\mathrm{BzCl}$ and the selectivity for 2,5-DMBP at $393 \mathrm{~K}$ using 1:20 $(\mathrm{BzCl}$ : p-xyl). 


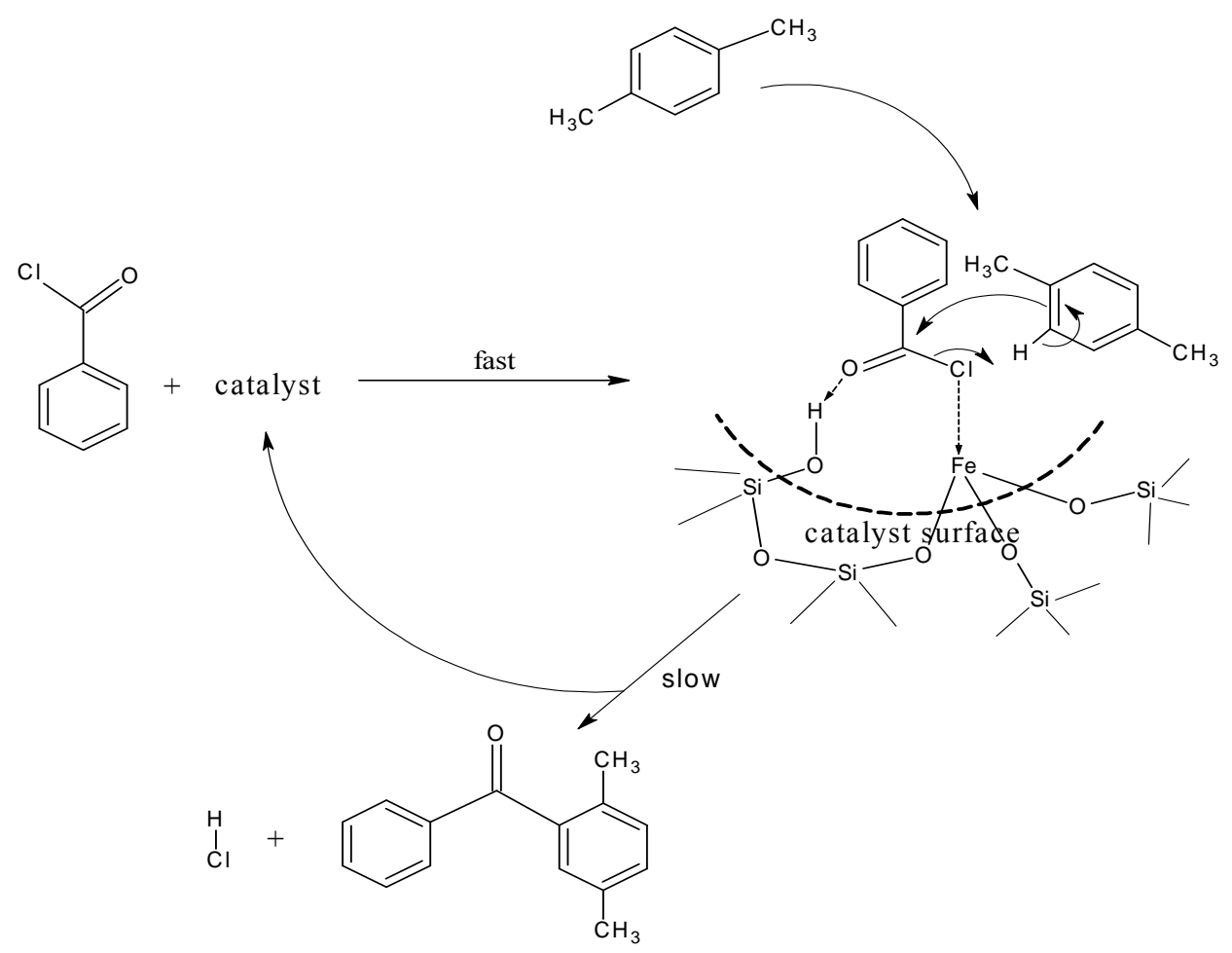

Fig. (10). The proposed reaction mechanism for the benzoylation of $p$-xyl over RHA-xFe (x = 5, 10, 15 and 20 wt.\%).

lyst decreased due to leaching.

It must now be reemphasized that during the reusability tests of the catalyst, the selectivity for the 2,5-DMBP did not change significantly, i.e. maintained at ca. 90\% (Table 5). This implies that although there was some leaching of the Fe centres, the efficiency in terms of the selectivity of the heterogeneous catalyst was not affected during its reuse. It can therefore, be concluded that the catalysts can be reused without loss of selectivity but with some loss in its efficiency. Further work is in progress to overcome the leaching and improve efficiency.

\section{Proposed Reaction Mechanism}

Generally, acylation of aromatics takes place by electrophilic aromatic substitution [44]. A schematic diagram of the proposed reaction mechanism for the benzoylation of $p$-xyl is presented in Fig. 10. The proposed mechanism involves the formation of an adsorbed electrophilic transition species (fast step), which is generated from the benzoyl chloride by interaction with the surface of the catalyst as shown. This reacts with $p$-xyl resulting in the formation of 2,5dimethylbenzophenone (a bimolecular slow step) with the simultaneous elimination of $\mathrm{HCl}$.

\section{CONCLUSIONS}

Heterogeneous catalysts with different Fe loadings using rice husk silica as the support were successfully prepared at room temperature. These catalysts showed high activity in the benzoylation of $p$-xyl. It also showed a high selectivity towards 2,5-DMBP. RHA-10Fe was found to be the best in terms of high conversion and selectivity. It showed $98.4 \%$ conversion of $\mathrm{BzCl}$ and $88.9 \%$ selectivity towards 2,5DMBP under the optimum conditions. The advantages of
RHA-Fe catalyst are that it can be prepared easily and does not need to be calcined after preparation. The catalyst can be reused although there is a decrease in the catalytic activity, which is related to the leaching of the Fe by benzoyl chloride and benzoic acid.

\section{ACKNOWLEDGEMENT}

The authors would like to thank the Malaysian Government and Universiti Sains Malaysia for the Research University grant (Ac. No: 1001/PKIMIA/814019). The University of Khartoum, Sudan, is acknowledged for providing a scholarship to I.A. Hassan.

\section{REFERENCES}

[1] Geneste, P.; Finiels, A. Aromatic Benzoylation. In Catalysts for Fine Chemical Synthesis Microporous and Mesoporous Solid Catalysts; Derouane, E.G., Ed.; John Wiley \& Sons Ltd: Chichester, 2006; vol. 4, pp. 95-104

[2] Yadav, G.D.; Asthana, N.S.; Kamble, V.S. Friedel-Crafts benzoylation of $p$-xylene over clay supported catalysts: novelty of cesium substituted dodecatungstophosphoric acid on K-10 clay. Appl. Catal. A., 2003, 240, 53-69.

[3] Olah, G.A. Friedel-Crafts and Related Reactions, vol 1; Wiley Interscience: New York, 1963.

[4] Choudhary, V.R.; Jana, S.K. Benzylation of benzene by benzyl chloride over Fe-, Zn-, Ga- and In-modified ZSM-5 type zeolite catalysts. Appl. Catal. A., 2002, 224, 51-62.

[5] Laidlaw, P.; Bethell, D.; Brown, S.M.; Hutchings, G.J. Benzoylation of substituted arenes using $\mathrm{Zn}$-and Fe-exchanged zeolites as catalysts. J. Mol. Catal. A: Chem., 2001, 174, 187-191.

[6] Izumi, Y.; Ogawa, M.; Urabe, K. Alkali metal salts and ammonium salts of Keggin-type heteropolyacids as solid acid catalysts for liquid-phase Friedel-Crafts reactions. Appl. Catal. A., 1995, 132, $127-$ 140 .

[7] Jacob, B.; Sugunan, S.; Singh, A.P. Selective benzoylation of $o$ xylene to 3,4-dimethylbenzophenone using various zeolite catalysts. J. Mol. Catal. A. Chem., 1999, 139, 43-53. 
[8] Choudhary, V.R.; Jana, S.K.; Patil, N.S.; Bhargava, S.K. FriedelCrafts type benzylation and benzoylation of aromatic compounds over $\mathrm{H} \beta$ zeolite modified by oxides or chlorides of gallium and indium. Microporous. Mesoporous. Mater., 2003, 57, 21-35.

[9] Suja, H.; Deepa, C.S.; Rani, K.S.; Sugunan, S. Liquid phase benzoylation of arenes over iron promoted sulphated zirconia. Appl. Catal., A, 2002, 230, 233-243.

[10] Choudhary, V.R.; Jana, S.K. Benzoylation of benzene and substituted benzenes by benzoyl chloride over $\mathrm{In}_{2} \mathrm{O}_{3} / \mathrm{Si}$-MCM-41 catalyst. J. Mol. Catal. A. Chem., 2002, 184, 247-255.

[11] Kalapathy, U.; Proctor, A.; Schultz, J. A simple method for production of pure silica from rice hull ash. Bioresour. Technol., 2000, 73, 257-262.

[12] Mekhemer, G.A.H.; Abd-Allah, H.M.M.; Mansour, S.A.A. Surface characterization of silica-supported cobalt oxide catalysts. Colloids Surf. A., 1999, 160, 251-259.

[13] Chang, F.W.; Hsiao, T.J.; Chung, S.W.; Lo, J.J. Nickel supported on rice husk ash - activity and selectivity in $\mathrm{CO}_{2}$ methanation. Appl. Catal. A., 1997, 164, 225-236.

[14] Chang, F.W.; Tsay, M.T.; Liang, S.P. Hydrogenation of $\mathrm{CO}_{2}$ over nickel catalysts supported on rice husk ash prepared by ion exchange. Appl. Catal. A., 2001, 209, 217-227.

[15] Chang, F.W.; Kuo, M.S.; Tsay, M.T.; Hsieh, M.C. Hydrogenation of $\mathrm{CO}_{2}$ over nickel catalysts on rice husk ash-alumina prepared by incipient wetness impregnation. Appl. Catal. A., 2003, 247, 309320 .

[16] Chang, F.W.; Yang, H.C.; Roselin, L.S.; Kuo, W.Y. Ethanol dehydrogenation over copper catalysts on rice husk ash prepared by ion exchange. Appl. Catal. A., 2006, 304, 30-39.

[17] Adam, F.; Chua, J.H. The adsorption of palmytic acid on rice husk ash chemically modified with $\mathrm{Al}(\mathrm{III})$ ion using the sol-gel technique. J. Colloid. Interface. Sci., 2004, 280, 55-61.

[18] Adam, F.; Kandasamy, K.; Balakrishnan, S. Iron incorporated heterogeneous catalyst from rice husk ash. J. Colloid. Interface. Sci., 2006, 304,137-143.

[19] Adam, F.; Andas, J. Amino benzoic acid modified silica—An improved catalyst for the mono-substituted product in the benzylation of toluene with benzyl chloride. J. Colloid. Interface. Sci., 2007, 311, 135-143.

[20] Ahmed, A.E.; Adam, F. The benzylation of benzene using aluminium, gallium and iron incorporated silica from rice husk ash. Microporous. Mesoporous. Mater., 2009, 118, 35-43.

[21] Adam, F.; Ahmed, A.E. The benzylation of xylenes using heterogeneous catalysts from rice husk ash silica modified with gallium, indium and iron. Chem. Eng. J., 2008, 145, 328-334.

[22] Ahmed, A.E.; Adam, F. Indium incorporated silica from rice husk and its catalytic activity. Microporous. Mesoporous. Mater., 2007,103, 284-295.

[23] Yalçin, N.; Sevinç, V. Studies on silica obtained from rice husk. Ceram. Int., 2001, 27, 219-224.

[24] Della, V.P.; Kühn, I.; Hotza, D. Rice husk ash as an alternate source for active silica production. Mater. Lett., 2002, 57, 818-821.

[25] Kalapathy, U.; Proctor, A.; Shultz, J. Silica xerogels from rice hull ash: structure, density and mechanical strength as affected by gelation $\mathrm{pH}$ and silica concentration. J. Chem. Technol. Biotechnol., 2000, 75, 464-468.

[26] Liou, T.H. Preparation and characterization of nano structured silica from rice husk. Mater, Sci. Eng. A., 2004, 364, 313-323.
[27] Chandrasekhar, S.; Pramada, P.N.; Praveen, L. Effect of organic acid treatment on the properties of rice husk silica. J. Mater. Sci., 2005, 40, 6535-6544.

[28] Burns, D.T; Tungkananurak, K.; Jadsadapattarakul, D. Semi-Micro Preparation and Characterization of Bonded Phase ODS-Silica Prepared from Rice Husk Silica. Microchim. Acta., 2006, 154, 81-85.

[29] Maxim, N. Ph.D. Thesis, Eindhoven University of Technology, 2002.

[30] Gregg, S.J.; Sing, K.S.W. Adsorption, Surface Area and Porosity, 2nd ed.; Academic Press Inc: London, 1982.

[31] Vinu, A.; Sawant, D.P.; Ariga, K.; Hartmann, M.; Halligudi, S.B. Benzylation of benzene and other aromatics by benzyl chloride over mesoporous AlSBA-15 catalysts. Microporous. Mesoporous. Mater., 2005, 80, 195-203.

[32] Tao, Y.; Kanoh, H.; Abrams, L.; Kaneko, K. Mesopore-modified zeolites: preparation, characterization, and applications. Chem. Rev., 2006, 106, 896-910.

[33] Iler, R.K. The Chemistry of Silica; John Willy \& Sons Inc: USA, 1979.

[34] Bachari, K.; Millet, J.M.M.; Benaïchouba, B.; Cherifi, O.; Figueras, F. Benzylation of benzene by benzyl chloride over iron mesoporous molecular sieves materials. J. Catal., 2004, 221, 5561.

[35] Samanta, S.; Giri, S.; Sastry, P.U.; Mal, N.K.; Manna, A.; Bhaumik, A. Synthesis and characterization of iron-rich highly ordered mesoporous Fe-MCM-41. Ind. Eng. Chem. Res., 2003, 42, 30123018.

[36] Szegedi, Á.; Kónya, Z.; Méhn, D.; Solymár, E.; Pál-Borbély, G.; Horváth, Z.E.; Biró, L.P.; Kiricsi, I.. Spherical mesoporous MCM41 materials containing transition metals: synthesis and characterization. Appl. Catal. A., 2004, 272, 257-266.

[37] Pais da Silva, M.I.; Lins da Silva, F.; Téllez S, C.A. FT-infrared band analysis and temperature programmed dessorption for the $\mathrm{Y}$, $\mathrm{L}$ and ferrierite zeolites. Spectrochim. Acta. Part. A., 2002, 58, 3159-3166.

[38] Chakraborty, B.; Viswanathan, B. Surface acidity of MCM-41 by in situ IR studies of pyridine adsorption. Catal. Today., 1999, 49, 253-260.

[39] Akçay, M. The surface acidity and characterization of $\mathrm{Fe}$ montmorillonite probed by in situ FT-IR spectroscopy of adsorbed pyridine. Appl. Catal. A., 2005, 294, 156-160.

[40] Yadav, G.D.; Asthana, N.S.; Kamble, V.S. Cesium-substituted dodecatungstophosphoric acid on K-10 clay for benzoylation of anisole with benzoyl chloride. J. Catal., 2003, 217, 88-99.

[41] Selvakumar, S.; Singh, A.P. Benzoylation of anisole over silicotungstic acid modified mesoporous alumina. Catal. Lett., 2009 128, 363-372.

[42] Corma, A. From microporous to mesoporous molecular sieve materials and their use in catalysis. Chem. Rev., 1997, 97, 2373-2420.

[43] Arends, I.W.C.E.; Sheldon, R.A. Activities and stabilities of heterogeneous catalysts in selective liquid phase oxidations: recent developments. Appl. Catal, A., 2001, 212,175-187.

[44] Olah, G.A.; Kobayashi, S. Aromatic substitution. XXIX. FriedelCrafts Acylation of benzene and toluene with substituted acyl halides. effect of substituents on substrate and positional selectivity. J. Am. Chem. Soc., 1971, 93, 6964-6967.

(C) Adam et al.; Licensee Bentham Open.

This is an open access article licensed under the terms of the Creative Commons Attribution Non-Commercial License (http://creativecommons.org/licenses/by-nc/3.0/) which permits unrestricted, non-commercial use, distribution and reproduction in any medium, provided the work is properly cited. 